\title{
3. Milieutherapie und Ansätze aus der Soteria - Wie gestalte ich das Umfeld therapeutisch?
}

\author{
Eric Hahn und Albert Diefenbacher
}

Die psychiatrische Behandlung fußt auf drei grundlegenden Therapiesäulen, die insbesondere bei stationären Behandlungen schwerer psychischer Erkrankungen individuell an die Symptomatik und Situation angepasst werden. Neben der auf der biologischen Psychiatrie beruhenden Pharmakotherapie, sind dies die Psychotherapie und die Soziotherapie. Letztere umfasst die Behandlung von psychischen Störungen durch die gezielte Beeinflussung von Milieufaktoren, u.a. durch Strukturierung des Tagesablaufes, Interaktionen zwischen Patienten, Behandlern und Angehörigen, aber auch ergo-, kunst-, musik- und arbeitstherapeutische Behandlungsansätze.

Beispielhaft für eine primär auf das therapeutische Milieu abzielende Behandlung stellt Ihnen dieses Kapitel zunächst die Grundeinstellungen der Soteria - einem milieutherapeutischen Ansatz für akute psychotische Störungen vor. Anschließend werden Möglichkeiten der Einbindung von „Soteria-Elementen“ in die Versorgungsrealität auf allgemeinpsychiatrischen Akutstationen, insbesondere für Patienten mit Schizophrenien, aufgezeigt.

\section{Was bedeutet Milieutherapie?}

Milieutherapie beschreibt eine gezielte Gestaltung der Umgebungsvariablen und des Tagesablaufes, um einen gemeinsamen therapeutischen Prozess zu ermöglichen.

Der im Englischen oft synonym verwendete Begriff der „social-therapy“ beinhaltete dabei die Ausweitung von Prinzipien von Gruppentherapien auf Aspekte des sozialen Milieus mit dem Ziel, die psychische Gesundheit der Patienten zu fördern. Ein Hauptziel der Milieutherapie stellt die Aufrechterhaltung einer positi- 
ven und unterstützenden Atmosphäre durch einfühlsame und transparente Beziehungen zu den Behandlern in Kombination mit einer Vielzahl möglicher Einzelmaßnahmen dar. Fragen Sie bei Entlassung Ihre Patienten, was während des Aufenthaltes geholfen habe, werden Sie nicht selten Antworten wie: „der Austausch mit Mitpatienten“, „vertrauensvolle Gespräche auf Augenhöhe“, „nicht die einzige mit den Problemen zu sein“ und „Verständnis in der Gemeinschaft" hören.

\section{Die therapeutische Gemeinschaft}

Verständnis und nicht wertende Kontaktmöglichkeiten als Wirkprinzipien wurden mit dem Konzept der therapeutischen Gemeinschaft bereits in den 4oer-Jahren, zunächst in Großbritannien, beschrieben. Ausgehend von der humanistisch geprägten Weltanschauung, dass Interaktionen in einer Gemeinschaft therapeutisch wirksam sind, sollten auch die Beziehungen zwischen Patienten untereinander und dem therapeutischen Team reflektiert und bewusst gestaltet werden. Hintergrund war die Suche nach Alternativen der gängigen psychiatrischen Behandlung in psychiatrischen Großkrankenhäusern bzw. „Anstalten“. Eine spezifische antipsychotische wirksame Medikation, die eine der Voraussetzungen der Psychiatriereform und der Gemeindepsychiatrie war, stand erst Jahre später, frühestens ab 1953 zur Verfügung.

Die Grundideen der therapeutischen Gemeinschaft basieren auf gruppenpsychoanalytischen, lerntheoretischen und sozialpsychologischen Konzepten, wobei Werte wie Offenheit, Übernahme von Eigenverantwortung mit dem Ziel einer individuellen Entwicklung im Vertrauen auf die Gemeinschaft geteilt werden. Viele heute selbstverständliche Haltungen in klinisch-stationären, teilstationären und komplementären Behandlungsangeboten wie Partnerschaft aller Teammitglieder, gegenseitiges Lernen, Ressourcenorientierung und Fokussierung auf die individuellen Bedürfnisse von Patienten wurden bereits bei der Konzeptualisierung der therapeutischen Gemeinschaft vertreten.

\section{Milieu in psychiatrischen Kliniken}

Stationen und Tageskliniken mit spezialisierten sucht- oder psychotherapeutischen Angeboten, aber auch zur Behandlung neurokognitiver Störungen im Alter nutzen heutzutage Konzepte der Milieutherapie und der therapeutischen Gemeinschaft. Aber auch das Milieu in allgemeinpsychiatrischen Abteilungen, mit einer durchschnittlichen Verweildauer von 25 Tagen, unterscheidet sich gegenüber dem Milieu auf somatischen Stationen:

Im Gegensatz zur somatischen Station werden psychiatrische Patienten ihren Tag nicht primär im Zimmer verbringen, um bspw. auf die nächste Untersuchung oder die Visite zu warten, sondern sie werden angehalten, an therapeutischen Aktivitäten nach einem individuell festgelegten Tagesplan teilzunehmen. Einige zentrale Elemente therapeutischer Gemeinschaften wie Gemeinschaftsaktivitäten (z.B. Gruppenvisiten am Morgen, gemeinsame Mahlzeiten im Gemeinschaftsraum statt im Zimmer, Wochenbesprechung zur Verteilung gemein- 
schaftlicher Aufgaben), multiprofessionelle Team-Sitzungen und Gruppentherapien (z.B. Psychoedukationsgruppen, Depressionsgruppen, Essgruppen) werden Ihnen im klinischen Alltag immer wieder begegnen. Ein therapeutisches Milieu kann so Kommunikationsmöglichkeiten und gegenseitiges Verständnis fördern, um mit den Patienten ein Gefühl von Miteinander, einen Perspektivenwechsel und schließlich wieder eine Außenorientierung zu erreichen.

\section{Kritik an der psychiatrischen Krankenhausbehandlung}

In der Vergangenheit wurde das psychiatrische Milieu, insbesondere auf geschlossenen psychiatrischen Stationen mit dem Schwerpunkt auf Behandlung schizophrener Psychosen, ab Mitte der Goer-Jahre zunehmend kritisiert. Die breite Verfügbarkeit und die symptomatische Wirksamkeit hochpotenter Antipsychotika auf Positivsymptome mit den Leitsubstanzen Haloperidol (Europa) und Perphenazin (USA) bedingten teilweise eine einseitige Betonung von Medikation in der Behandlung von Schizophrenien. Behandlungsziele waren Symptomreduktion und medikamentöse Compliance, wobei im Vergleich zur aktuellen Pharmakotherapie oft sehr hohe Dosen üblich waren. Noch in den 8oer-Jahren lag die mittlere Dosis bei $20-25 \mathrm{mg} / \mathrm{d}$ Haloperidol, wobei Patienten bei Hochdosistherapien mit Tagesdosen von über $100 \mathrm{mg}$ Haloperidol behandelt wurden. Nebenwirkungen waren eine deutliche Sedierung, sekundär verstärkte Negativsymptome und extrapyramidal motorische Symptome.

Weitere Kritikpunkte bildeten die geringen Personalstellen, die langen Aufenthaltszeiten (2/3 der Patienten > 1 Jahr) in oft außerhalb von Städten und Gemeinden gelegenen großen psychiatrischen Krankenhäusern, wobei die Patienten in Vielbettzimmern oder Schlafsälen untergebracht waren. Die psychiatrischen Kliniken galten als hierarchisch strukturiert, Patienten wurden in Entscheidungsfragen kaum involviert und Selbstverantwortung oder Selbsthilfe spielten eine untergeordnete Rolle.

Vor diesem Hintergrund formierte sich die sogenannte AntipsychiatrieBewegung, die sich in ihrer Kritik vornehmlich gegen das Milieu in der damaligen institutionellen Psychiatrie, aber auch gegen die vorherrschenden Krankheitszuschreibungen und Behandlungsangebote richtete. Die Kritik richtet sich hauptsächlich gegen die Diagnose der Schizophrenie mit der, insbesondere in den USA, ein Großteil der Patienten in psychiatrischen Kliniken diagnostiziert war. Antipsychiatrische Gegenentwürfe zu einer biologisch-genetischen bedingten Genese, sahen die Ursache einer schizophrenen Symptomatik ausschließlich in den vorherrschenden gesellschaftlichen Strukturen, die durch das Milieu und die Kommunikationsstile in psychiatrischen Kliniken noch verstärkt würden. Ein Verdienst dieser kritischen Auseinandersetzung war, dass die AntipsychiatrieBewegung wichtige Impulse zu einer Verbesserung der Qualität und Reform der psychiatrischen Versorgung gegeben hat. So ist ab den 7oer-Jahren das Konzept der Soteria entstanden. Es fordert zu gleichwertigen Beziehungen zwischen Behandlern und Patienten auf und beeinflusst insbesondere die Behandlung schizophrener Psychosen. 


\section{Soteria als milieutherapeutische Alternative}

Mit der „Soteria“ (griechisch: Rettung, Befreiung, Bewahrung) wollte der US-amerikanische Psychiater Loren Mosher Anfang der 70er-Jahre eine offene gemeindezentrierte wohngemeinschaftsartige Einrichtung außerhalb von tradierten Klinikstrukturen etablieren (Mosher 2011; Mosher et al. 2004).

Die Soteria war speziell für die Behandlung jüngerer, akut an schizophrenen Psychosen erkrankten Menschen entwickelt worden und verbindet Ansätze der therapeutischen Gemeinschaft mit einem familienartigen Milieu, in dem die Ziele der Patienten im Vordergrund stehen. Soteria bedeutete einen bewussten Gegenentwurf zur klassischen Krankenhausbehandlung. Diese, so die Annahme, sei geprägt durch eine von der Normalität weiter entfremdete, unstete und für die Patienten unberechenbare und teils durch Gewalt, Zwang und Angst charakterisierte Atmosphäre. Dazu würden ein häufiger Wechsel des Personals, unpersönliche und unübersichtliche Räumlichkeiten sowie ein Herausfallen aus gewohnten Umweltzusammenhängen oder Regeln des Zusammenlebens beitragen. Zudem werde das Selbstwertgefühl der stationären Patienten durch innere und äußere Stigmatisierung eines Aufenthaltes in der Psychiatrie, mögliche Zwangsmaßnahmen, Gewalt durch Mitpatienten und eine autoritäre Entscheidungsfindung ohne Mitsprachegelegenheit der Patienten weiter vermindert. In einer Zeit der angesprochenen antipsychotischen Hochdosistherapien mit teils erheblichen und irreversiblen Nebenwirkungen (insb. Spätdyskinesien) wurde die Soteria von Patienten, die auch Klienten genannt wurden, und Angehörigen tatsächlich als eine Befreiung verstanden.

Als Gegenperspektive zur vorherrschenden Behandlung in geschützten Großstationen kann eine Forderung nach einer weitgehend medikamentenfreien, aktiven mitmenschlichen 1:1-Begleitung während der psychotischen Krise („beingwith“) durch ein festes Betreuerteam mit tragenden Beziehungsangeboten in einer familienähnlichen Wohnatmosphäre (max. 1o Klienten) als revolutionäre Herausforderung etablierter Strukturen nachvollzogen werden. Eine im Rahmen der Soteria mit der Zeit akzeptierte antipsychotische Niedrigdosierung ist durchaus mit den heutzutage empfohlenen Niedrigdosierungen vergleichbar.

Die Behandlung wurde in drei Phasen gegliedert.

In der Akutphase hat die Stimuluskontrolle durch eine reizarme, sicherheitsvermittelnde Umgebung bei kontinuierlicher ruhiger und haltender („,containing“) Anwesenheit der Bezugsperson oberste Priorität. Durch einfühlendes Verständnis im Sinne einer Patienten-zentrierten Sichtweise wurde dem subjektiven Verständnis des psychotischen Erlebens wertungsfrei durch das „Dabei-Sein“ Raum gegeben. Als entscheidender Wirkfaktor gilt die authentische zwischenmenschliche Begegnung. Medikamentös wurden bei Bedarf und Wunsch des Patienten Benzodiazepine und niedrigdosierte Antipsychotika eingesetzt.

In der Stabilisierungs- und Aktivierungsphase sollten insbesondere soziale und lebenspraktische Fertigkeiten aufrechterhalten werden. Vor dem Hintergrund, dass bei Schizophrenien automatische Handlungs- und Wahrnehmungsschemata sowie unbewusst eingeübte soziale Skripte wieder in ihre Bestandteile zerfallen, ist das Trainieren dieser Handlungen in einem zwischenmenschlichen alltagsnahen 
Kontext von großer Bedeutung. Diese Orientierung auf Alltagsrelevanz und lebenspraktische Fertigkeiten durch ein in der Gemeinschaft sozial eingebettetes Lernen steht im Gegensatz zu den meisten stationären Therapieprogrammen, die dazu neigen, komplexe Handlungen aufzuspalten und in einem künstlichen Setting zu trainieren. Im Vordergrund der zweiten Phase stehen demnach Gemeinschaftsaktivitäten wie Kochen, Einkaufen, Tischdecken und Zimmer putzen oder Haus aufräumen. Die Zuteilung der Aufgaben erfolgt in gemeinschaftlichen Diskussionen, um die Selbstverantwortlichkeit und Selbstwirksamkeit zu stärken, wobei die mitwohnenden Betreuer ebenso Haushaltsaufgaben übernehmen.

Die Wendung zur Außenwelt und Entlassungsvorbereitung: Angehörige und nachbetreuende Personen werden noch stärker in die Behandlung und Entlassungsplanung eingebunden. Die Patienten werden explizit ermutigt, ihr soziales Leben in der gewohnten Alltagsumgebung stattfinden zu lassen. Das Erleben in der psychotischen Krise soll in die Lebenskontinuität und das soziale Netzwerk des Betroffenen eingefügt werden. Die Soteria selbst bleibt ein Teil dieser Alltagsumgebung, sodass Kontakte mit den Soteria-Betreuern und Mitbewohnern auch nach der Entlassung aufrechterhalten werden sollen.

Tabelle 12 fasst für Sie wichtige Kriterien für eine Soteria nach L. Mosher zusammen.

Tab. 12 Kriterien für eine Soteria basierend auf L. Mosher (Soteria-Netzwerk o.J.)
1. Setting
kleines, gemeindenahes, klinikexternes, auf Freiwilligkeit beruhendes Setting in einer heimartigen Umgebung, das Sicherheit und Schutz gewährt
2. soziales Umfeld respektvolles, konsistentes und für den Patienten voraussagbares soziales Umfeld; Sicherheit gewährend, individuelle Stimuluskontrolle
3. soziale Struktur Erhaltung von persönlicher Entscheidungsfreiheit und Autonomie, Förderung von gegenseitigen Beziehungen, geringe Rollendifferenzierung
4. Betreuer neben den bekannten Berufsgruppen ist ein Einbezug von Angehörigen, geschulten Laien und ehemaligen Klienten möglich, berufsbegleitende Supervision aller Betreuenden; Stetigkeit des Personals
5. Beziehungen zwischenmenschliche Beziehungen bilden das zentrale Element der Soteria, Akzeptanz und offener phänomenologischer Zugang zu psychotischem Erleben, aktives Dabeisein und gemeinsames Handeln, Verzicht auf Jargon und Etikettie- rung.
6. Therapie alle Aktivitäten werden als potenziell „therapeutisch“ eingestuft. Therapien orien- tieren sich am Alltagsleben und sind ressourcenorientiert, „Mit-Tun“ und „Mit- Sein“ als Wirkfaktoren; keine formellen Therapiesitzungen mit Ausnahme von Familieninterventionen
7. Medikation keine oder nur niedrig dosierte antipsychotische Medikation, um eine zu starke Dämpfung oder Unterdrückung von Emotionen zu vermeiden
8. Aufenthaltsdauer ausreichend lang, um persönliche Beziehungen zu entwickeln und das psychoti- sche Erleben in die persönliche Lebensgeschichte einzuordnen 


\section{Weiterentwicklung des Soteria-Konzepts im deutschsprachigen Raum}

Der Soteria-Ansatz wurde im deutschsprachigen Raum, kurz nach Schließung der Soteria von L. Mosher in Kalifornien, durch den Psychiater Luc Ciompi 1984 zunächst in Bern eingeführt. Er erweiterte das Verständnis der Wirkprinzipien der Soteria bei Schizophrenie durch sein Konzept der Affektlogik. Sein Ausgangspunkt ist, dass Fühlen und Denken, also Affekt und Logik, bei allen psychischen Leistungen zusammenwirken und sich gegenseitig beeinflussen. Eine zu starke emotionale Belastung beispielsweise durch anhaltenden Streit oder Verlusterlebnisse wäre, falls die entsprechende Vulnerabilität vorliegt, der Ausgangspunkt für das Übergehen in eine psychotische Krise, mit entsprechenden Denk- und Wahrnehmungsstörungen.

Aufgabe der Akutbehandlung in der Soteria sei es daher zunächst, eine emotionale Entspannung zu schaffen, worunter sich nach der Affektlogik auch das Denken und Wahrnehmen stabilisieren sollten. Die Kernpunkte der Soteria wie eine tragende, reizgeschützte Atmosphäre in einem möglichst normalen therapeutischen Milieu, basierend auf transparenter und behutsamer mitmenschlicher Begleitung durch wenige Bezugspersonen wurden beibehalten. Ciompi erweiterte das Konzept durch die Einführung eines „weichen Zimmers“ in dem reizabgeschirmt und mittels tragender 1:1-Betreuung die Patienten durch akute psychotische Krisen begleitet werden konnten.

Wichtig war eine personelle Kontinuität mit Schichten, die bis zu 48 Stunden andauerten. In allen Phasen sollte eine enge Zusammenarbeit mit den Angehörigen und wichtigen Bezugspersonen erfolgen, wobei Informationen in Bezug auf die Erkrankung und Behandlungsziele den Betroffenen, Angehörigen und Betreuenden gleichartig und auch im Trialog kommuniziert werden sollten.

In den letzten 15 Jahren wurden auch in Deutschland mehrere Soteria-Einrichtungen etabliert, zunächst in Zwiefalten und bei München. Gleichzeitig werden in allgemeinpsychiatrischen Abteilungen vermehrt milieutherapeutische Konzepte verwendet. Die zunächst antipsychiatrisch geprägten Soteria-Konzepte wurden im Sinne einer Synthese weiter adaptiert, sodass eine primär medikamentenfreie Behandlung schizophrener Psychosen aktuell kaum noch vertreten wird. Diese Entwicklung wurde unterstützt durch die Verfügbarkeit von nebenwirkungsärmeren und auf Negativsymptome wirkende Antipsychotika, die zudem in deutlich niedrigeren Dosierungen verordnet werden.

Im Sinne von Partizipation und Authentizität werden Soteria-Bewohner frühzeitig in alle Entscheidungsprozesse einschließlich einer Medikation einbezogen, wobei als Ziel eine möglichst nebenwirkungsarme antipsychotische Pharmakotherapie und die selbstverantwortliche Einnahme der Medikation als Rückfallprophylaxe definiert wird. Als wichtiger Erfolgsindikator von Soteria-Einrichtungen werden interessanterweise eine letztlich höhere Langzeitadhärenz und 
geringere Wiederaufnahmeraten und damit langfristig geringere Behandlungskosten aufgeführt. In diesem Sinne ist auch die Forderung nach einer systematischen Nachbetreuung unter Einbeziehung des sozialen Netzes, mit Fokus auf Frühwarnsymptome und Bewältigungsstrategien, und einer medikamentösen Rückfallprophylaxe Teil der modernen Behandlungskonzepte vieler psychiatrischer Abteilungen.

Eine weitere Adaptation stellen sogenannte „Soteria-Stationen“ innerhalb psychiatrischer Kliniken dar, die von dem ursprünglich geforderten klinikfernen Milieu (z.B. einer ausgebauten Villa) abweichen. Eine weitere Einführung von SoteriaElementen in den gesamten Bereich der integrierten Versorgung psychotischer Störungen könnte so im Rahmen des gemeindepsychiatrischen Ansatzes ermöglicht werden.

\section{Leitlinien für (akutpsychiatrische) Stationen mit integrierten Soteria-Elementen}

Das Konzept der Soteria unterliegt bei den aktuellen baulichen und gesundheitsökonomischen Bedingungen deutlichen Einschränkungen, die einer stärkeren Verbreitung der klassischen Soteria entgegenstehen. Im Zuge der Psychiatriereform wurden psychiatrische Abteilungen bevorzugt in Allgemeinkrankenhäusern gegründet, sodass ein klinikexternes Setting kaum möglich ist. Die langen Schichten von 24-48 Stunden durch die Soteria-Betreuenden sind für die meisten Beschäftigten nicht durchführbar oder gewünscht. Die wenigen in Deutschland verfügbaren Soterias sind fast immer voll belegt. In Bezug auf das Patientenkollektiv werden zumeist jüngere Menschen mit akuten schizophrenen Psychosen aufgenommen. Patienten, die als akut selbst- oder fremdgefährdend eingeschätzt werden, und bei denen eine Bündnisfähigkeit bzw. Freiwilligkeit nicht gegeben ist, werden meist nicht in einer Soteria behandelt. Weitere Ausschlusskriterien können eine bestehende Abhängigkeitserkrankung, insbesondere durch Alkohol, Medikamente und Drogen darstellen. Selten notwendige Zwangsmaßnahmen finden in dem Milieu einer Soteria meist nicht statt.

Als eine Alternative konnten in etlichen psychiatrischen Kliniken sogenannte „Soteria-Elemente“ in den klinischen Alltag integriert werden. Zum einen bedeutet dies, dass diese Stationen offen geführt werden, wohnlicher umgebaut und ggf. verkleinert werden. Zum anderen ist ein Perspektivwechsel der Betreuenden, Angehörigen aber auch der Patienten notwendig. Soteria bedeutet auch Authentizität, solidarische Gemeinschaft und Übernahme von Selbstverantwortung bei allen Beteiligten, also auch durch die Patienten.

Bei Überlegungen zur Umsetzbarkeit des Soteria-Konzepts in den Alltag einer psychiatrischen Akut-Station wurden von V. Bonnet und W. Machleidt (SoteriaNetzwerk o.J.) einige Kriterien erarbeitet, die Sie als Anregungen zur Gestaltung des Stationsmilieus verstehen sollten. Eine durchaus kritisch reflektierende Anpassung dieser Konzepte an die Gegebenheiten und Machbarkeiten in Ihrer psychiatrischen Abteilung oder Station kann dabei ein sehr lohnenswertes Ziel darstellen. Dabei geht es uns weniger um die Idee, dass zukünftig möglichst viele Akutstationen den Beinamen „mit integrierten Soteria-Elementen“ tragen, als 
dass diese Elemente bei konsequenter Umsetzung das funktionelle Outcome der Patienten verbessern und gleichzeitig die Zufriedenheit bei Patienten und Behandlern steigern können.

Leitlinien und Kriterien für (akutpsychiatrische) Stationen mit integrierten Soteria-Elementen (SoteriaNetzwerk 0.J.)

\section{Rahmenbedingungen}

- konzeptuelle und personelle Kontinuität während der Behandlung

- Behandlung im multiprofessionellen Team

- Supervision/gemeinsame Fortbildungen im multiprofessionellen Team

- Bezugstherapeutensystem und Konstanz der Behandler bei Wiederaufnahme

- Weiterbehandlung im gemeindepsychiatrischen Versorgungssystem

Grundhaltung

- zwischenmenschliche Beziehung als primärer Bestandteil der Therapie

- Dabeisein („,being-with“) - durch den psychotischen Zustand begleiten

- „ganzheitliches Psychoseverständnis“ und Akzeptanz der Psychose-Erfahrung

- Individualisierung des Behandlungsprozesses mit Fokus auf das subjektive Erleben

- positive Erwartungshaltung in Bezug auf das erreichbare Funktionsniveau

- Förderung von Resilienz durch Anknüpfen an individuelle Ressourcen

- Stärken der Eigenverantwortung und eigenen Ziele

Milieutherapeutische Merkmale

- möglichst geringe Patientenzahl

- persönliches Milieu durch individuelle wohnlichere Gestaltung der Station

- Station als Begegnungs- und Kommunikationsraum

- offene Stationstür

- klinische Hierarchie minimiert bei prinzipieller Gleichwertigkeit der Teammitglieder

- Aufgabenteilung und Mitbestimmung bei der Gestaltung des stationären Alltags

- Einbeziehung der Angehörigen in den therapeutischen Prozess

Therapeutische Interventionen

- Aufnahme durch Bezugstherapeuten ohne vorgeschaltete zentrale Aufnahme

- an den Bedürfnissen des Patienten orientiertes Aufnahmegespräch

- Orientierung gebende Hilfestellungen

- Aufbau einer vertrauensvollen Beziehung mit Regulierung von Nähe und Distanz

- unterstützende, tragende Haltung des Teams

- empathisches Verstehen, auch der psychotischen Symptomatik

- kontinuierliche Behandlungsplanung gemeinsam mit dem Patienten

- behutsamer Umgang mit Psychopharmaka

- selbständiger eigenverantwortlicher Umgang mit Medikamenten

- Möglichkeit der Begleitung im weichen Zimmer und 1:1-Kontakt bei Bedarf

- biographische Integration des psychotischen Erlebens

- Gruppenzusammenkünfte zum Leben und Lernen in der Gemeinschaft

- kontinuierliches Einbeziehen des sozialen Umfelds

- bedürfnisorientierte familiäre Unterstützung 


\section{Literaturempfehlung}

Aebi E, Ciompi L, Hansen H (Hrsg.) (2013) Soteria im Gespräch: Über eine alternative Schizophreniebehandlung. Erweiterte Neuauflage. Paranus-Verlag Neumünster

Ciompi L, Hoffmann H, Broccard M (Hrsg.) (2011) Wie wirkt Soteria? Eine atypische Psychosenbehandlung kritisch durchleuchtet. Carl-Auer Verlag Heidelberg

Mosher LR (2001) Soteria-California and its successors: Therapeutic ingredients. In: Ciompi L, Hoffmann H, Broccard M (Hrsg.) Wie wirkt Soteria? - Ein atypische Psychosenbehandlung kritisch durchleuchtet. Carl-Auer Verlag Heidelberg

Mosher LR, Burti L (1992) Psychiatrie in der Gemeinde. Grundlagen und Praxis. Psychiatrie-Verlag Bonn

Mosher LR, Hendrix V, Fort D (2004) Soteria: Through Madness to Deliverance. Xlibris-Verlag USA

Nischk D, Merz P, Rusch I (2014) Aktuelles aus der Soteria - Die Förderung lebenspraktischer und sozialer Fertigkeiten von Menschen mit Schizophrenien aus phänomenologischer Sicht. Psychiatrische Praxis 41(01), 45-49

Soteria-Netzwerk (о.J.) Kriterien für Soteria-Einrichtungen. URL: http://www.soteria-netzwerk.de/krit-einrich.htm (abgerufen am 18.02.2015)

Soteria-Netzwerk (о.).) Kriterien für (akutpsychiatrische) Stationen mit integrierten Soteria-Elementen. URL: http://www.soteria-netzwerk.de/krit-station.htm (abgerufen am 18.02.2015) 